\title{
Improved Outcomes with Arthroscopic Bone Marrow Aspirate Concentrate and Cartilage-Derived Matrix Implantation versus Chondroplasty for the Treatment of Focal Chondral Defects of the Knee Joint: A Retrospective Case Series
}

\author{
Iciar M. Dávila Castrodad, M.D., Matthew J. Kraeutler, M.D., Sydney M. Fasulo, M.D., \\ Anthony Festa, M.D., Vincent K. McInerney, M.D., and Anthony J. Scillia, M.D.
}

\begin{abstract}
Purpose: To compare the outcomes of patients undergoing treatment of focal chondral defects (FCDs) of the knee joint with chondroplasty versus bone marrow aspirate concentrate (BMAC) and cartilage-derived matrix (CDM) implantation. Methods: A retrospective chart review was performed for patients diagnosed with Outerbridge grade 3-4 FCDs. Patients were included if they were treated arthroscopically with BMAC/CDM implantation or chondroplasty alone between March 2016 and May 2019 and had more than 1-year follow-up. Postoperative outcomes included the visual analog scale (VAS) for pain; University of California, Los Angeles (UCLA) activity scores; Knee Outcome Survey (KOS) Activities of Daily Living (ADL) and Sports subscores; postoperative corticosteroid or hyaluronic acid injections; subsequent surgeries; and conversion to total knee arthroplasty. Results: A total of 98 patients were identified with a mean follow-up in BMAC/CDM of 24 months (range 13-4l months) and in chondroplasty of 44 months (range 34-55 months). A subanalysis was performed to control for significant differences in age, which yielded 39 patients, ages 40-60 years. Within the subanalysis group, mean VAS scores were significantly lower in the BMAC/CDM group $(1.7$ vs $4.4 ; P=.005)$ and mean UCLA scores were significantly greater $(7.1$ vs $5.0 ; P=.002)$. Mean improvement in VAS and UCLA scores were similar between the BMAC/CDM and chondroplasty groups $(-3.7$ vs $-1.3 ; P=.71,1.9$ vs $0.1 ; P=.14$, respectively). Mean KOS $\mathrm{ADL}$ and Sports subscores were significantly greater among patients in the BMAC/CDM group $(87 \%$ vs $55 \% ; P=.001$, $71 \%$ vs $41 \% ; P=.002$, respectively). There were no differences in postoperative injections, subsequent surgeries, or conversion to total knee arthroplasty between the BMAC/CDM and chondroplasty groups. Conclusions: Patients with grade 3-4 FCDs of the knee had improved postoperative outcomes when treated with BMAC/CDM implantation versus chondroplasty alone, as evidenced by a significant improvement in VAS and UCLA scores and significantly greater postoperative KOS ADL, and KOS Sport subscores. Level of Evidence: IV, therapeutic case series.
\end{abstract}

$\mathbf{F}$ ull-thickness focal chondral defects (FCDs) of the knee joint are common and can lead to symptoms of a degenerative joint such as pain, swelling, and joint dysfunction. ${ }^{1,2}$ If conservative treatments fail, multiple surgical procedures may be offered for these lesions, including chondroplasty, microfracture/drilling,

From the Department of Orthopaedic Surgery, St. Joseph's University Medical Center, Paterson (I.M.D.C., M.J.K., S.M.F., A.F., V.K.M., A.J.S.); and Seton Hall University, South Orange (A.F., V.K.M., A.J.S.), New Jersey, U.S.A.

The authors report the following potential conflicts of interest or sources of funding: V.K.M. reports board membership, New Jersey Orthopaedic Society, MD Advantage Board of Trustees; and stock/stock options from Topical Gear. A.J.S. reports board membership, AAOS, American Orthopaedic Society for Sports Medicine, and New Jersey Orthopaedic Society; consultant for Mitek; stock/stock options from Biomet, CONMED Linvatec, Johnson \& Johnson, Pfizer, Smith $\theta$ Nephew, and Stryker; and research support from Isto-biologics. Full ICMJE author disclosure forms are available for this article online, as supplementary material. osteochondral autograft or allograft transplantation, and autologous chondrocyte implantation, among others. $^{3-8}$ Chondroplasty involves debriding unstable cartilage flaps with the use of a mechanical shaver or radiofrequency device to stabilize chondral lesions and prevent these lesions from growing in size. ${ }^{9}$

Received June 24, 2021; accepted October 26, 2021

Address correspondence to Anthony J. Scillia, M.D., Department of Orthopaedic Surgery, St. Joseph's University Medical Center, Hackensack Meridian School of Medicine at Seton Hall University, 703 Main St., Paterson, NJ 07503. E-mail: anthonyjscillia@gmail.com

(C) 2021 THE AUTHORS. Published by Elsevier Inc. on behalf of the Arthroscopy Association of North America. This is an open access article under the CC BY-NC-ND license (http://creativecommons.org/licenses/by-nc-nd/4.0/). 2666-061X/21898

https://doi.org/10.1016/j.asmr.2021.10.018 
In recent years, there has been an increased level of interest in the use of biologic therapies for the treatment of FCDs. Examples include platelet-rich plasma, bone marrow aspirate concentrate (BMAC), and mesenchymal stem cells. ${ }^{10-13}$ These therapies offer antiinflammatory and regenerative properties, which make them attractive options for clinicians. One recently proposed biologic therapy includes the use of BMAC with a dehydrated, micronized allogeneic cartilagederived matrix scaffold (CDM) for grade 4 FCDs. ${ }^{14,15}$ The purpose of this study was to compare the outcomes of patients undergoing treatment of FCDs of the knee joint with chondroplasty versus BMAC and CDM implantation. The authors hypothesized that the BMAC/CDM group would demonstrate better postsurgical outcomes than the chondroplasty group.

\section{Methods}

\section{Patient Selection}

Following institutional review board approval, a retrospective chart review was performed for patients diagnosed with grade 3-4 FCDs, as defined by the Outerbridge Classification system. Patients were included if they were treated arthroscopically with BMAC/CDM implantation by the senior author (A.J.S.) or chondroplasty alone performed by 2 different sports fellowship-trained surgeons, between March 2016 and May 2019, had greater than $2 \mathrm{~cm}$ of joint space on radiographs, and had more than 1-year follow-up. Patients with lesions greater than $5 \mathrm{~cm}^{2}$ were excluded. Patient demographics including age, sex, and body mass index (BMI) were collected. The location and the number of FCDs were documented as well.

\section{Surgical Interventions}

The BMAC and CDM implantation surgery was performed by the senior author (A.J.S.) when grade 4 FCDs were identified. This technique, which has been previously published, was a bone marrow aspiration of $60 \mathrm{~mL}$ from the contralateral hip and subsequent centrifugation (Magellan; Isto Biologics, Hopkinton, $\mathrm{MA}$ ), yielding $7 \mathrm{~mL}$ of BMAC. ${ }^{14}$ This was followed by diagnostic arthroscopy to identify and address any additional pathology. Additional procedures were performed when indicated and included: loose body removal, percutaneous skeletal fixation (Subchondroplasty; Zimmer Biomet, Warsaw, IN), ligament reconstruction, osteotomy, lateral release, and partial meniscectomy. The FCD was then grafted with a mixture of BMAC and CDM (BioCartilage; Arthrex, Inc., Naples, FL). Lastly, the remaining BMAC was used to inject the knee intra-articularly.

The chondroplasty procedure was performed by 2 other surgeons (A.F. and V.K.M.) when grade III and IV FCDs were identified. The surgery consisted of diagnostic arthroscopy to identify the presence of additional pathology and concomitantly address these. Additional procedures included loose body removal, partial meniscectomy, cyst debridement or aspiration, lateral release, and synovectomy. Subsequently, the FCD underwent chondroplasty to stabilize the articular cartilage.

\section{Variables}

Postoperative outcomes included pain intensity, functional scores, and complications. Pain intensity was measured by using the visual analog scale (VAS) for pain and was collected pre- and postoperatively. Functional outcomes were assessed with the University of California Los Angeles (UCLA) activity score, Knee Outcome Survey (KOS) Activities of Daily Living (ADL), and KOS Sports subscores. The UCLA scores were collected pre- and postoperatively whereas the KOS ADL and Sports subscores were collected postoperatively. Postoperative injections with corticosteroid or hyaluronic acid for persistent pain were documented as well. Complications were defined as wound-, implant- or hardware-related complications; subsequent surgeries; and conversion to total knee arthroplasty (TKA).

\section{Data Analysis}

Using the $G^{*}$ Power 3.1.9.4 software, a post hoc power analysis included all participants and was performed with the study parameters of 0.8 effect size and $\alpha=$ 0.05 yielding a power of $96 \%$. Student $t$ tests were used to compare the average age, BMI, pain, and functional scores between the 2 groups. $\chi^{2}$ tests were used to compare sex, the number and location of FCDs, the number of patients who received postoperative injections, the number of patients who underwent subsequent surgeries, and the number of patients who underwent conversion to TKA. A $P$ value $<.05$ was considered statistically significant. All statistical analyses were performed using SPSS, version 22 (IBM Corp., Armonk, NY).

\section{Results}

\section{Patients and Demographics}

A total of 98 patients were identified for the study, 37 of whom were treated with BMAC/CDM implantation whereas 61 were treated with chondroplasty alone. The mean follow-up in BMAC/CDM was 24 months (range 13-41 months), significantly shorter than in chondroplasty, which was 44 months (range 34-55 months; $P<.001)$. There were no significant differences in sex between the BMAC/CDM and chondroplasty groups ( $46 \%$ vs $56 \%$ females; $P=.35$ ) or BMI ( 29 vs $30 \mathrm{~kg} / \mathrm{m}^{2}$; $P=.14$ ) (Table 1). However, patients in the BMAC/ CDM group were significantly younger (40 vs 62 years; 
Table 1. Patient Demographics and Postoperative Outcomes Stratified by Type of Intervention

\begin{tabular}{|c|c|c|c|}
\hline Variables & Chondroplasty $(\mathrm{n}=61)$ & BMAC/CDM Implantation $(\mathrm{n}=37)$ & $P$ Value \\
\hline \multicolumn{4}{|l|}{ Patient demographics } \\
\hline Age, $y^{*}$ & $62(11)$ & $40(16)$ & $<.001$ \\
\hline \multicolumn{4}{|l|}{$\operatorname{Sex}^{\dagger}$} \\
\hline $\mathrm{BMI}^{*}$ & $30(5)$ & $29(7)$ & .14 \\
\hline \multicolumn{4}{|l|}{ FCD characteristics ${ }^{\dagger}$} \\
\hline \multicolumn{4}{|l|}{ Location of FCD } \\
\hline Patella & $25(41)$ & $12(32)$ & .40 \\
\hline Trochlea & $17(28)$ & $6(16)$ & .19 \\
\hline LTP & $6(10)$ & $3(8)$ & .77 \\
\hline MTP & $17(28)$ & $2(5)$ & .01 \\
\hline Number of multifocal FCD lesions & $36(59)$ & $6(16)$ & $<.001$ \\
\hline \multicolumn{4}{|l|}{ Concomitant procedures ${ }^{\dagger}$} \\
\hline Revision ACL reconstruction & $0(0)$ & $2(5)$ & .14 \\
\hline MPFL reconstruction & $1(2)$ & $5(14)$ & .03 \\
\hline Meniscal repair & $0(0)$ & $1(3)$ & .38 \\
\hline Loose body removal & $9(15)$ & $6(16)$ & .85 \\
\hline Plica excision & $0(0)$ & $2(5)$ & .14 \\
\hline Baker's cyst excision & $2(3)$ & $4(11)$ & .20 \\
\hline Lateral release & $0(0)$ & $6(16)$ & .002 \\
\hline IT band lengthening & $0(0)$ & $1(3)$ & .38 \\
\hline Tibial tubercle osteotomy & $0(0)$ & $1(3)$ & .38 \\
\hline Distal femoral osteotomy & $0(0)$ & $1(3)$ & .38 \\
\hline Follow-up, mo* & $44(5)$ & $24(6)$ & $<.001$ \\
\hline \multicolumn{4}{|l|}{ Postoperative outcomes } \\
\hline Complications $^{\dagger}$ & $0(0)$ & $4(11)$ & .01 \\
\hline Cellulitis & 0 & 2 & \\
\hline Cyst recurrence & 0 & 1 & \\
\hline Wound dehiscence & 0 & 1 & \\
\hline Subsequent surgeries ${ }^{\dagger}$ & $4(7)$ & $3(8)$ & .77 \\
\hline Revision chondroplasty & 4 & 0 & \\
\hline Open cyst excision & 0 & 1 & \\
\hline MUA & 0 & 1 & \\
\hline Removal of hardware & 0 & 1 & \\
\hline Conversion to $\mathrm{TKA}^{\dagger}$ & $11(18)$ & $1(3)$ & .03 \\
\hline
\end{tabular}

ACL, anterior cruciate ligament; ADL, Activities of Daily Living; BMAC, bone marrow aspirate concentrate; BMI, body mass index; FCD, focal chondral defect; IT, iliotibial; KOS, Knee Outcome Score; LFC, lateral femoral condyle; LTP, lateral tibial plateau; MFC, medial femoral condyle; MPFL, medial patellofemoral ligament; MTP, medial tibial plateau; MUA, manipulation under anesthesia; TKA, total knee arthroplasty; UCLA, University of California, Los Angeles; VAS, visual analog score.

*The values are given as the mean (standard deviation).

${ }^{\dagger}$ The values are given as the number (percent); Differences in VAS and UCLA scores are equal to the differences between preoperative and postoperative values.

$P<.001)$. The majority of FCDs were found in the medial femoral condyle and the patella. Significantly fewer multifocal lesions were found in the BMAC/CDM group (6 vs $36 ; P<.001$ ).
A subanalysis was performed to control for the significant age discrepancy between the groups and identified patients ages 40 to 60 years and yielded 39 patients (16 BMAC/CDM, 23 chondroplasty) with 
Table 2. Subanalysis Patient Demographics and Postoperative Outcomes Stratified by Type of Intervention (Patients Between the Ages of 40 and 60 Years)

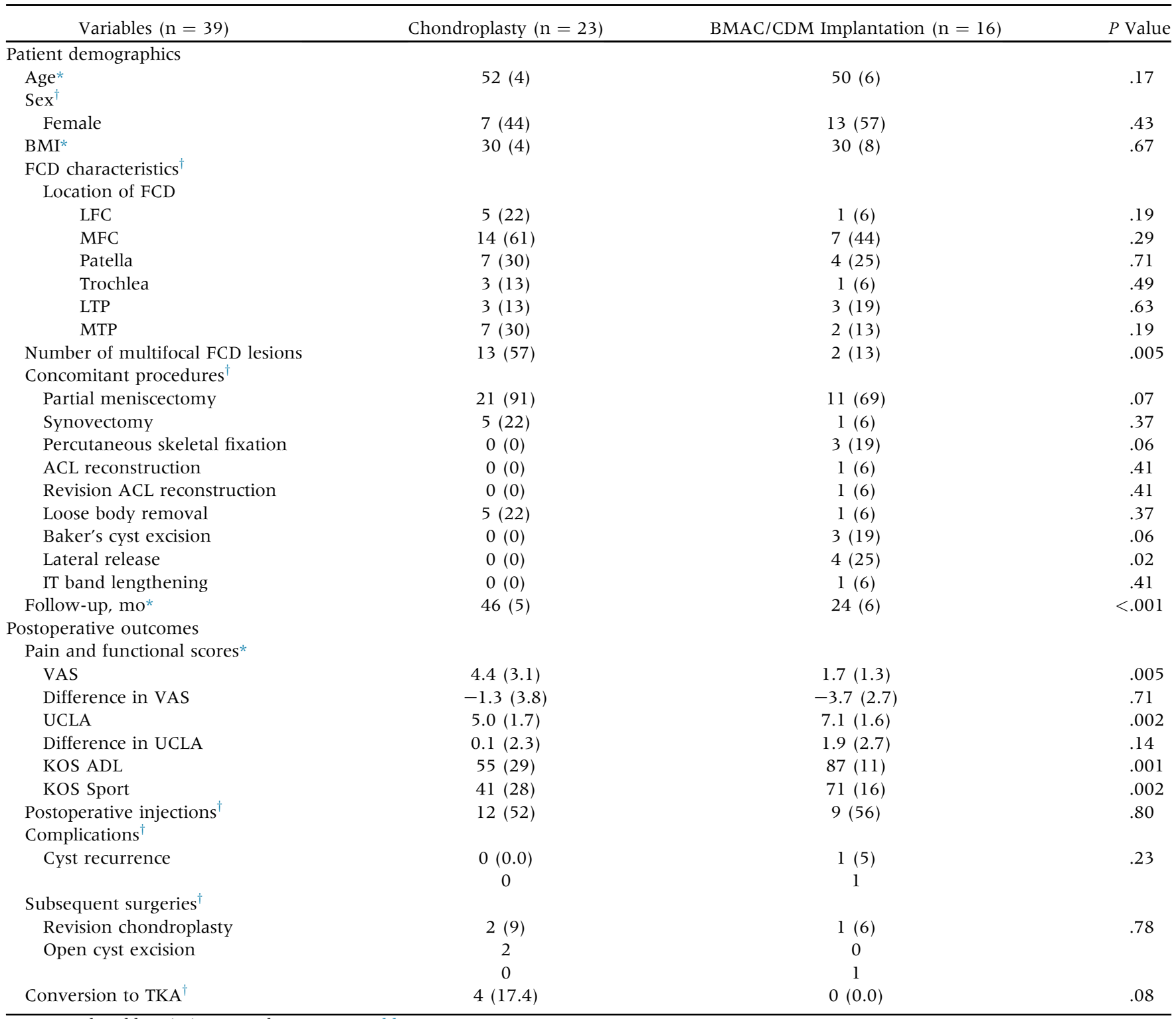

NOTE. The abbreviations are the same as Table 1.

*The values are given as the mean (standard deviation).s

${ }^{\dagger}$ The values are given as the number (percent); Differences in VAS and UCLA scores are equal to the differences between preoperative and postoperative values.

similar age ( 50 vs 52 years; $P=.17$ ), sex $(57 \%$ vs $44 \%$ female; $P=.43)$, and BMI ( $\left.30 \mathrm{vs} 30 \mathrm{~kg} / \mathrm{m}^{2} ; P=.67\right)$ (Table 2). Most FCDs were located in the medial femoral condyle and the patella. After subanalysis, there remained significantly fewer multifocal lesions ( 3 vs 13; $P=.005$ ) and shorter follow-up (24 vs 46 months; $P<.001)$ in the BMAC/CDM group.

\section{Patient-Reported Outcome Measures}

Among the entire cohort, preoperative mean VAS (5.22 vs $5.74 ; P=.39$ ) and UCLA (4.81 vs $4.42 ; P=.40$ ) did not differ between BMAC/CDM and chondroplasty groups. Postoperative outcome measures differed; VAS scores were significantly lower among the BMAC/CDM group (1.2 vs 3.4, $P<.001)$ and mean UCLA scores were significantly greater $(8.0$ vs $5.2, P<.001)$. Significantly greater mean improvements were noted in VAS $(-4.0 \mathrm{vs}-2.1, P=.13)$ and UCLA scores (2.9 vs $0.2, P=.001)$ for the BMAC/CDM group. Mean KOS $\mathrm{ADL}$ and Sport subscores were significantly greater among patients in the BMAC/CDM group (91\% vs $66 \%, P<.001 ; 80 \%$ vs $55 \%, P=.001$, respectively). 
Within the subanalysis group, VAS scores remained significantly lower among the BMAC/CDM group (1.7 vs $4.4, P=.005)$ and mean UCLA scores remained significantly greater (7.1 vs $5.0, P=.002)$. Mean improvements in VAS and UCLA scores were similar for the BMAC/CDM and chondroplasty groups $(-3.7$ vs $-1.3, P=.71 ; 1.9$ vs $0.1, P=.14$, respectively). Mean KOS ADL and Sport subscores were significantly greater among patients in the BMAC/CDM group $(87 \%$ vs $55 \%, P=.001 ; 71 \%$ vs $41 \%, P=.002$, respectively).

\section{Complications}

Among the entire cohort, more minor complications occurred in the BMAC/CDM group (4 vs $0, P=.01$ ) including stitch abscess and/or cellulitis (which resolved with oral antibiotics and wound care) and a Baker's cyst recurrence after surgery. There were no significant differences in the number of patients receiving postoperative injections for persistent pain (10 vs $24, P=$ $.21)$. Three patients $(8 \%)$ in the BMAC/CDM group underwent subsequent surgeries compared with 4 patients $(7 \%)$ in the chondroplasty group $(P=.77)$. Fewer BMAC/CDM patients were converted to TKA (1 vs $11, P=.03)$.

Within the subanalysis group, there were no differences in the percentage of patients receiving postoperative injections ( $44 \%$ vs $48 \%, P=.80$ ), subsequent surgeries $(6 \%$ vs $9 \% ; P=.78)$, or conversions to TKA $(0 \%$ vs $17 \% ; P=.08)$ between the BMAC/CDM and chondroplasty groups.

\section{Discussion}

In this study, we found there were improved outcomes in patients undergoing BMAC/CDM compared with chondroplasty. These findings are consistent with a systematic review that included 13 clinical studies that all demonstrated clinical improvement in FCDs treated with various BMAC augmented therapies; however, the literature presently available is sparce and of lower scientific quality. ${ }^{11}$ Although the overall mean age of the chondroplasty patients in our study was significantly greater than the BMAC/CDM group, we performed a subanalysis on patients aged between 40 and 60 years at the time of surgery. Within this subanalysis group, we found significantly better VAS, UCLA, and KOS ADL and Sports subscores in the BMAC/CDM group as well as a trend toward significantly better outcomes in this group with respect to rate of conversion to TKA.

While chondroplasty is a procedure used to stabilize potentially unstable chondral lesions and does not restore the lost articular cartilage, ${ }^{16}$ BMAC contains mesenchymal stem cells (MSCs) and growth factors to reduce inflammation and potentially regenerate hyaline cartilage, ${ }^{17}$ although this is yet to be proven in vivo. BioCartilage is an extracellular matrix scaffold developed from dehydrated allograft cartilage that contains type II collagen, proteoglycans, and cartilaginous growth factors. ${ }^{18}$ Thus, the concept of using the combination of BMAC and BioCartilage is to provide a structural scaffold on which MSCs from the BMAC may regenerate hyaline-like cartilage. While BioCartilage previously has been used in conjunction with microfracture to stimulate the subchondral bone marrow elements, ${ }^{19}$ the use of BioCartilage with BMAC provides the same advantages while avoiding the disadvantages of microfracture such as damage to the subchondral bone and osteocyte necrosis. ${ }^{4}$

The improved outcomes of BMAC/CDM treatment should be weighed against the disadvantages of this procedure. This procedure takes longer than chondroplasty and requires bone marrow aspirate (BMA) to be harvested from the iliac crest. Although complications from BMA harvest are rare, they do occur and may include persistent donor-site pain, lateral femoral cutaneous neuralgia, superficial hematoma or infection, and fracture of the anterior superior iliac spine. ${ }^{20}$ The concentration of progenitor cells and other nonstem cell types varies widely based on patient age, anatomic location of harvest, and processing technique, which may affect MSC behavior and growth factor content. ${ }^{21}$ Lastly, BMAC treatment is not covered by insurance companies and may result in additional outof-pocket costs for patients or place the financial onus on the surgical facility to cover the cost of this additional procedure.

\section{Limitations}

The limitations of this study should be noted. This was a retrospective study, with short-term follow-up, in which the concomitant procedures performed differed between the 2 groups making it challenging to decipher patient outcomes based solely on the cartilage treatments performed. Although a subanalysis was performed to compare patients of similar ages, this resulted in a limited sample size and no power analysis was performed. The follow-up time still differed significantly between the 2 groups, which may have affected the rate of conversion to TKA. Finally, the KOS ADL and Sports subscores were not collected preoperatively, and therefore it was not possible to compare the mean improvement in these scores between the 2 groups.

\section{Conclusions}

Patients with grade 3-4 FCDs of the knee had improved postoperative outcomes when treated with BMAC/CDM implantation versus chondroplasty alone, as evidenced by a significant improvement in VAS and UCLA scores and significantly greater postoperative KOS ADL, and KOS Sport subscores. 


\section{References}

1. Chubinskaya S, Haudenschild D, Gasser S, Stannard J, Krettek C, Borrelli J Jr. Articular cartilage injury and potential remedies. J Orthop Trauma 2015;29:S37-S52 (suppl 12).

2. Ciccotti MC, Kraeutler MJ, Austin LS, et al. The prevalence of articular cartilage changes in the knee joint in patients undergoing arthroscopy for meniscal pathology. Arthroscopy 2012;28:1437-1444.

3. Cole BJ, Pascual-Garrido C, Grumet RC. Surgical management of articular cartilage defects in the knee. J Bone Joint Surg Am 2009;91:1778-1790.

4. Kraeutler MJ, Aliberti GM, Scillia AJ, McCarty EC, Mulcahey MK. Microfracture versus drilling of articular cartilage defects: A systematic review of the basic science evidence. Orthop J Sports Med 2020;8:2325967120945313.

5. Kraeutler MJ, Belk JW, Purcell JM, McCarty EC. Microfracture versus autologous chondrocyte implantation for articular cartilage lesions in the knee: A systematic review of 5-year outcomes. Am J Sports Med 2018;46:995-999.

6. Schrock JB, Kraeutler MJ, Houck DA, McQueen MB, McCarty EC. A cost-effectiveness analysis of surgical treatment modalities for chondral lesions of the knee: Microfracture, osteochondral autograft transplantation, and autologous chondrocyte implantation. Orthop J Sports Med 2017;5:2325967117704634.

7. Schuette HB, Kraeutler MJ, McCarty EC. Matrix-assisted autologous chondrocyte transplantation in the knee: A systematic review of mid- to long-term clinical outcomes. Orthop J Sports Med 2017; 5:2325967117709250.

8. Schuette HB, Kraeutler MJ, Schrock JB, McCarty EC. Primary autologous chondrocyte implantation of the knee versus autologous chondrocyte implantation after failed marrow stimulation: A systematic review. Am J Sports Med 2021;49:2536-2541.

9. Spahn G, Kahl E, Mückley T, Hofmann GO, Klinger HM. Arthroscopic knee chondroplasty using a bipolar radiofrequency-based device compared to mechanical shaver: Results of a prospective, randomized, controlled study. Knee Surg Sports Traumatol Arthrosc 2008; 16:565-573.

10. Boffa A, Previtali D, Altamura SA, Zaffagnini S, Candrian C, Filardo G. Platelet-rich plasma augmentation to microfracture provides a limited benefit for the treatment of cartilage lesions: A meta-analysis. Orthop J Sports Med 2020;8:2325967120910504.

11. Cavinatto L, Hinckel BB, Tomlinson RE, Gupta S, Farr J, Bartolozzi AR. The role of bone marrow aspirate concentrate for the treatment of focal chondral lesions of the knee: A systematic review and critical analysis of animal and clinical studies. Arthroscopy 2019;35: 1860-1877.

12. Kraeutler MJ, Chahla J, LaPrade RF, Pascual-Garrido C. Biologic options for articular cartilage wear (platelet-rich plasma, stem cells, bone marrow aspirate concentrate). Clin Sports Med 2017;36:457-468.

13. Ryu DJ, Jeon YS, Park JS, Bae GC, Kim JS, Kim MK. Comparison of bone marrow aspirate concentrate and allogenic human umbilical cord blood derived mesenchymal stem cell implantation on chondral defect of the knee: Assessment of clinical and magnetic resonance imaging outcomes at 2-year follow-up. Cell Transplant 2020;29:963689720943581.

14. Dávila Castrodad IM, Mease SJ, Werheim E, McInerney VK, Scillia AJ. Arthroscopic chondral defect repair with extracellular matrix scaffold and bone marrow aspirate concentrate. Arthrosc Tech 2020;9: el24l-el247.

15. Dávila Castrodad IM, Simone ES, Kurowicki J, et al. Improved short-term outcomes of osteochondral lesions of the knee following arthroscopic treatment with bone marrow aspirate concentrate and cartilage-derived matrix. Arthrosc Sports Med Rehabil 2021;2:e477-e484.

16. Kosy JD, Schranz PJ, Toms AD, Eyres KS, Mandalia VI. The use of radiofrequency energy for arthroscopic chondroplasty in the knee. Arthroscopy 2011;27:695-703.

17. Gobbi A, Whyte GP. One-stage cartilage repair using a hyaluronic acid-based scaffold with activated bone marrow-derived mesenchymal stem cells compared with microfracture: Five-year follow-up. Am J Sports Med 2016;44:2846-2854.

18. Hirahara AM, Mueller KW Jr. BioCartilage: A new biomaterial to treat chondral lesions. Sports Med Arthrosc Rev 2015;23:143-148.

19. Cole BJ, Haunschild ED, Carter T, et al. Clinically significant outcomes following the treatment of focal cartilage defects of the knee with microfracture augmentation using cartilage allograft extracellular matrix: A multicenter prospective study. Arthroscopy 2021;37:1512-1521.

20. Hernigou P, Desroches A, Queinnec S, et al. Morbidity of graft harvesting versus bone marrow aspirate in cell regenerative therapy. Int Orthop 2014;38:1855-1860.

21. Lamplot JD, Rodeo SA, Brophy RH. A practical guide for the current use of biologic therapies in sports medicine. Am J Sports Med 2020;48:488-503. 